DO-TH 93-11

(August 1993)

\title{
CP-Violation in Bosonic Sector of SM with two Higgs Doublets
}

\author{
G. Cvetič \\ Inst. für Physik, Universität Dortmund, 44221 Dortmund, Germany
}

\begin{abstract}
We investigate CP-violation effects in the bosonic sector of the Standard Model (SM) with two Higgs doublets. First we calculate the mass eigenstates of the physical neutral Higgses for small but nonzero CP-violation parameter $\xi_{*}$, and then a "forward-backward" asymmetry $\mathcal{A}_{f b}$ for the decay $H \rightarrow W^{+} W^{-} Z$ that would be a signal of CP-violation. Although the effects are in general small $\left(\mathcal{A}_{f b}=\Gamma_{f b} / \Gamma \sim \mathcal{O}\left(10^{-3}\right)\right), \mathcal{A}_{f b}$ turns out to be a rather clean signal of $\mathrm{CP}$-violation, since neither the $\mathrm{CP}$-conserving final state interactions nor the direct production background events contribute to $\Gamma_{f b}$. The CKM-type CP-violation effects that could in principle also contribute to $\mathcal{A}_{f b}$ are negligible. The nonzero $\mathcal{A}_{f b}$ could possibly be detected at some later stage at the LHC or SSC.
\end{abstract}


The Standard Model (SM) with two Higgs doublets and CP-violation in the bosonic sector has recently drawn a lot of attention [1], especially because it could give an explanation of the baryogenesis [2, 3], unlike the CP-violation originating from the Cabibbo-Kobayashi-Maskawa (CKM) matrix of the SM.

The most general gauge invariant potential for two Higgs doublets $\Phi_{i}(i=1,2)$ with $Y=1$ which induces only suppressed flavor-changing neutral currents (FCNC's) is «4

$$
\begin{aligned}
V\left(\Phi_{1}, \Phi_{2}\right)= & \lambda_{1}\left(\Phi_{1}^{\dagger} \Phi_{1}-v_{1}^{2}\right)^{2}+\lambda_{2}\left(\Phi_{2}^{\dagger} \Phi_{2}-v_{2}^{2}\right)^{2} \\
& +\lambda_{3}\left[\left(\Phi_{1}^{\dagger} \Phi_{1}-v_{1}^{2}\right)+\left(\Phi_{2}^{\dagger} \Phi_{2}-v_{2}^{2}\right)\right]^{2}+\lambda_{4}\left[\left(\Phi_{1}^{\dagger} \Phi_{1}\right)\left(\Phi_{2}^{\dagger} \Phi_{2}\right)-\left(\Phi_{1}^{\dagger} \Phi_{2}\right)\left(\Phi_{2}^{\dagger} \Phi_{1}\right)\right] \\
& +\lambda_{5}\left[\operatorname{Re}\left(\Phi_{1}^{\dagger} \Phi_{2}\right)-v_{1} v_{2} \cos \xi\right]^{2}+\lambda_{6}\left[\operatorname{Im}\left(\Phi_{1}^{\dagger} \Phi_{2}\right)-v_{1} v_{2} \sin \xi\right]^{2} .
\end{aligned}
$$

The potential spontaneously breaks $S U(2)_{L} \times U(1)_{Y}$ down to $U(1)_{E M}$. The fact that the discrete symmetry $\Phi_{1} \mapsto-\Phi_{1}$ is only softly violated (by terms of dimension two) guarantees that the FCNC's are not too large. The six parameters $\lambda_{i}$ are in general of the order $\left(M_{\text {scalar }} / v\right)^{2} \sim \mathcal{O}(1)$. The minimum of the potential is at

$$
\left\langle\Phi_{1}\right\rangle_{o}=\left(\begin{array}{c}
0 \\
v_{1}
\end{array}\right), \quad\left\langle\Phi_{2}\right\rangle_{o}=e^{i \xi}\left(\begin{array}{c}
0 \\
v_{2}
\end{array}\right), \quad\left(v_{1}^{2}+v_{2}^{2}=\frac{1}{2} v^{2}=\frac{1}{2} 246^{2} G e V^{2}\right) .
$$

For $\xi_{*}\left(=\left(\lambda_{5}-\lambda_{6}\right) \xi\right)=0$, we have no CP-violation, and the neutral physical scalars $H_{+}^{o}, h_{+}^{o}, A_{-}^{o}$ have well-defined CP (equal to $+1,+1,-1$, respectively). These scalars and their masses are known (we use the notations of [4)

$$
\begin{aligned}
\left(\begin{array}{c}
H_{+}^{o} \\
h_{+}^{o}
\end{array}\right) & =\sqrt{2}\left(\begin{array}{rr}
\cos \alpha & \sin \alpha \\
-\sin \alpha & \cos \alpha
\end{array}\right)\left(\begin{array}{c}
\operatorname{Re} \Phi_{1}^{o}-v_{1} \\
\operatorname{Re} \Phi_{2}^{o}-v_{2}
\end{array}\right) \\
A_{-}^{o} & =\sqrt{2}\left(-\operatorname{Im} \Phi_{1}^{o} \sin \beta+\operatorname{Im} \Phi_{2}^{o} \cos \beta\right)
\end{aligned}
$$

where

$$
\begin{gathered}
\beta=\arctan \left(\frac{v_{2}}{v_{1}}\right), \quad \alpha=\frac{1}{2} \arctan \frac{2 \mathcal{M}_{12}}{\mathcal{M}_{11}-\mathcal{M}_{22}}, \quad \operatorname{sgn}(\sin 2 \alpha)=\operatorname{sgn}\left(\mathcal{M}_{12}\right) \\
\mathcal{M}=\left(\begin{array}{cc}
4 v_{1}^{2}\left(\lambda_{1}+\lambda_{3}\right)+v_{2}^{2} \lambda_{5} & \left(4 \lambda_{3}+\lambda_{5}\right) v_{1} v_{2} \\
\left(4 \lambda_{3}+\lambda_{5}\right) v_{1} v_{2} & 4 v_{2}^{2}\left(\lambda_{2}+\lambda_{3}\right)+v_{1}^{2} \lambda_{5}
\end{array}\right) \\
\left(\begin{array}{c}
M_{H^{o}}^{2} \\
M_{h^{\circ}}^{2}
\end{array}\right)=\frac{1}{2}\left[\mathcal{M}_{11}+\mathcal{M}_{22} \pm \sqrt{\left(\mathcal{M}_{11}-\mathcal{M}_{22}\right)^{2}+4 \mathcal{M}_{12}^{2}}\right], \quad M_{A^{o}}=\frac{1}{2} \lambda_{6} v^{2}
\end{gathered}
$$

On the other hand, in the case of $\xi_{*}=\left(\lambda_{5}-\lambda_{6}\right) \xi \neq 0$ we do have CP-violation. It is possible to find the physical scalar mass eigenstates in this case, if we make the expansion in powers 
of $\xi$ in the potential (1). Denoting the mass eigenstates $\left(H_{+}^{o}, h_{+}^{o}, A_{-}^{o}\right)$ of the $\xi_{*}=0$ case as $v_{(1)}(0), v_{(2)}(0), v_{(3)}(0)$, respectively, we obtain the three mass eigenstates $v_{(j)}\left(\xi_{*}\right)$ after some lengthy algebra:

$$
v_{(j)}\left(\xi_{*}\right)=U_{j k}\left(\xi_{*}\right) v_{(k)}(0)
$$

where $U\left(\xi_{*}\right)$ is a $3 \times 3$ orthogonal matrix:

$$
\begin{aligned}
U_{11}\left(\xi_{*}\right) & =1-\frac{1}{2} \xi_{*}^{2} \frac{\sin ^{2} \delta}{\left(x_{3}-x_{1}\right)^{2}}+\mathcal{O}\left(\left(\lambda_{5}-\lambda_{6}\right)^{2} \xi^{4}\right) \\
U_{22}\left(\xi_{*}\right) & =1-\frac{1}{2} \xi_{*}^{2} \frac{\cos ^{2} \delta}{\left(x_{3}-x_{2}\right)^{2}}+\mathcal{O}\left(\left(\lambda_{5}-\lambda_{6}\right)^{2} \xi^{4}\right) \\
U_{33}\left(\xi_{*}\right) & =1-\frac{1}{2} \xi^{2}\left[\frac{\sin ^{2} \delta}{\left(x_{3}-x_{1}\right)^{2}}+\frac{\cos ^{2} \delta}{\left(x_{3}-x_{2}\right)^{2}}\right]+\mathcal{O}\left(\left(\lambda_{5}-\lambda_{6}\right)^{2} \xi^{4}\right) \\
U_{12}\left(\xi_{*}\right) & =\xi^{2}\left(\lambda_{5}-\lambda_{6}\right) \frac{\left(\lambda_{5}-x_{1}\right) \sin (2 \delta)}{2\left(x_{1}-x_{2}\right)\left(x_{1}-x_{3}\right)}+\mathcal{O}\left(\left(\lambda_{5}-\lambda_{6}\right) \xi^{3}\right) \\
U_{21}\left(\xi_{*}\right) & =\xi^{2}\left(\lambda_{5}-\lambda_{6}\right) \frac{\left(\lambda_{5}-x_{2}\right) \sin (2 \delta)}{2\left(x_{2}-x_{1}\right)\left(x_{2}-x_{3}\right)}+\mathcal{O}\left(\left(\lambda_{5}-\lambda_{6}\right) \xi^{3}\right) \\
U_{31}\left(\xi_{*}\right) & =\xi_{*} \frac{\sin \delta}{\left(x_{1}-x_{3}\right)}+\mathcal{O}\left(\left(\lambda_{5}-\lambda_{6}\right) \xi^{3}\right)=-U_{13}\left(\xi_{*}\right) \\
U_{32}\left(\xi_{*}\right) & =\xi_{*} \frac{\cos \delta}{\left(x_{2}-x_{3}\right)}+\mathcal{O}\left(\left(\lambda_{5}-\lambda_{6}\right) \xi^{3}\right)=-U_{23}\left(\xi_{*}\right)
\end{aligned}
$$

where

$$
\xi_{*}=\left(\lambda_{5}-\lambda_{6}\right) \xi, \quad \delta=\beta+\alpha, \quad x_{j}=2 M_{j}^{2}(0) / v^{2} \quad(j=1,2,3) .
$$

Here, $M_{j}(0)(j=1,2,3)$ are masses of $v_{(j)}(0)$ (of eq. (4)). The masses of $M_{j}\left(\xi_{*}\right)$ of $v_{(j)}\left(\xi_{*}\right)$ differ from those of $\xi_{*}=0$ case only slightly (for $\xi \ll 1$ )

$$
M_{j}^{2}\left(\xi_{*}\right)=M_{j}^{2}(0)+\xi^{2}\left(\lambda_{5}-\lambda_{6}\right) \frac{v^{2}}{2} Y_{j}+\mathcal{O}\left(\left(\lambda_{5}-\lambda_{6}\right) \xi^{4}\right)
$$

where

$$
\begin{aligned}
Y_{1} & =\frac{\left(x_{1}-\lambda_{5}\right)}{\left(\lambda_{6}-x_{1}\right)} \sin ^{2} \delta, \quad Y_{2}=\frac{\left(x_{2}-\lambda_{5}\right)}{\left(\lambda_{6}-x_{2}\right)} \cos ^{2} \delta \\
Y_{3} & =1-\left(\lambda_{5}-\lambda_{6}\right)\left[\frac{\cos ^{2} \delta}{x_{2}-\lambda_{6}}+\frac{\sin ^{2} \delta}{x_{1}-\lambda_{6}}\right] .
\end{aligned}
$$

The quantities $\delta$ and $x_{j}$ are dimensionless, in general of order $\mathcal{O}(1)$. Note that the charged scalar sector remains unaffected by the introduction of $\xi_{*} \neq 0$.

Equation (5) tells us that the mass eigenstates of the neutral physical scalars are in general linear combinations of $C P=+1$ and $C P=-1$ components. This feature of CP-violation could be tested experimentally by looking at the decays of the (heavy) Higgs $H^{o}\left(=v_{(1)}\left(\xi_{*}\right)\right)$ to 
$W^{+} W^{-} Z$, as proposed within a more general context in ref. [5]. As argued there, in the unitary gauge, the decay amplitude $T_{+}$mediated by $W^{* \pm}$ and $Z^{*}$ exchange (Figs. 1a,b) would yield final states with $C P=+1$ (just like in the minimal $\mathrm{SM}$ ), while the decay amplitude $T_{-}$mediated by (neutral) physical scalars (Fig. 2) would yield final states with $C P=-1$ 円. On the other hand, the two-body decays $H^{o} \rightarrow W^{+} W^{-}, Z Z$ at the tree level would not test the mixed CPstructure of $H^{o}$, because the final state is an S-wave due to the coupling without derivatives $\left(L_{f i n}=0 \Rightarrow S_{f i n}=0 \Rightarrow C P\left(W^{+} W^{-}\right)=(-1)^{S_{f i n}}=+1=C P(Z Z)\right)$.

For the decay $H^{o} \rightarrow W^{+} W^{-} Z$, we can construct the following experimentally relevant "forward-backward" asymmetry width parameter $\Gamma_{f b}$ which would be a signal of this CPviolation

$$
\begin{gathered}
\Gamma_{f b}\left(H^{o} \rightarrow W^{+} W^{-} Z\right)= \\
{\left[\int_{0}^{+1} d(\cos \theta) \frac{d \Gamma\left(H^{o} \rightarrow W^{+} W^{-} Z\right)}{d(\cos \theta)}-\int_{-1}^{0} d(\cos \theta) \frac{d \Gamma\left(H^{o} \rightarrow W^{+} W^{-} Z\right)}{d(\cos \theta)}\right],}
\end{gathered}
$$

where $\theta$ is the angle between $\vec{p}_{W^{+}}$and $-\vec{p}_{Z}$ in the frame $\operatorname{CMS}\left(W^{+} W^{-}\right)$(Fig. 3), and the sum over the helicities of the final particles is implicitly assumed 1 .

Note that $\theta \mapsto \pi-\theta$ under the CP-transformation of the final state $W^{+} W^{-} Z$, and hence $\Gamma_{f b} \mapsto-\Gamma_{f b}$ if $H^{o}$ were a pure $C P=+1$ or $C P=-1$ state. Therefore, in the case of CPconservation we must have $\Gamma_{f b}=0$. Hence, $\Gamma_{f b} \neq 0$ is a signal of CP-violation. We can show in general, using the formalism of partial wave expansions of decay amplitudes [6], that $\Gamma_{f b}$ is an expression proportional to the interference terms of the $C P= \pm 1$ decay amplitudes

$$
\Gamma_{f b}\left(H^{o} \rightarrow W^{+} W^{-} Z\right) \propto \int d(\text { config.space })\left(T_{-}^{*} T_{+}+T_{-} T_{+}^{*}\right) \operatorname{sgn}(\cos \theta) .
$$

In the specific model at hand, we can check this by explicit calculation. The tree level transition amplitudes $T_{ \pm}$阳 in this case turn out to be

$$
T_{ \pm}=\epsilon^{\mu_{1}}\left(p_{1} h_{1}\right) \epsilon^{\mu_{2}}\left(p_{2} h_{2}\right) \epsilon^{\mu_{3}}\left(p_{3} h_{3}\right) \mathcal{F}_{\mu_{1} \mu_{2} \mu_{3}}^{( \pm)}\left(p_{1}, p_{2}, p_{3}\right)
$$

\footnotetext{
${ }^{1}$ The latter amplitude is zero if $\xi_{*}=0$, as expected.

${ }^{2}$ Note that $\Gamma_{f b}$ is in principle obtained by measuring the corresponding "forward-backward" difference $N_{f b}$ of the number of these decays: $\Gamma_{f b}=N_{f b} \frac{\Gamma\left(H^{o}\right)}{L \sigma}$, where $\Gamma\left(H^{o}\right)$ the total decay width, $L$ the integrated luminosity and $\sigma$ the production cross section for the (heavy) Higgs.

${ }^{3}$ Strictly speaking these are not tree level amplitudes, because the dominant final state interactions in the propagator are taken into account by nonzero widths of the mediating bosons. The amplitudes can be calculated in any $R_{\zeta}$-gauge, do not depend on the logitudinal parts of the propagators and have no $\zeta$-dependence.
} 
where $p_{j}$ and $h_{j}(j=1,2,3)$ denote momenta and helicities of $W^{+}, W^{-}$and $Z$, respectively, and

$$
\begin{aligned}
& \mathcal{F}^{(+)}=\mathcal{F}^{(+, 1)}+\mathcal{F}^{(+, 2)}+\mathcal{F}^{(+, 3)} \\
& \mathcal{F}_{\mu_{1} \mu_{2} \mu_{3}}^{(+, 1)}\left(p_{1} p_{2} p_{3}\right)=\frac{g^{2} \cos ^{2} \theta_{w} M_{Z}\left(U_{11} \cos \eta+U_{12} \sin \eta\right)}{\left[\left(p_{2}+p_{3}\right)^{2}-M_{W}^{2}+i \Gamma_{W} M_{W}\right]} \times \\
& {\left[2\left(p_{2 \mu_{3}} g_{\mu_{1} \mu_{2}}-p_{3 \mu_{2}} g_{\mu_{1} \mu_{3}}\right)-\left(p_{2}-p_{3}\right)_{\mu_{1}} g_{\mu_{2} \mu_{3}}-\frac{\sin ^{2} \theta_{w}}{\cos ^{2} \theta_{w}}\left(p_{1}+p_{2}+p_{3}\right)_{\mu_{1}} g_{\mu_{2} \mu_{3}}\right] \text {, }} \\
& \mathcal{F}_{\mu_{1} \mu_{2} \mu_{3}}^{(+, 2)}\left(p_{1} p_{2} p_{3}\right)=-\mathcal{F}_{\mu_{2} \mu_{1} \mu_{3}}^{(+, 1)}\left(p_{2} p_{1} p_{3}\right) \\
& \mathcal{F}_{\mu_{1} \mu_{2} \mu_{3}}^{(+, 3)}\left(p_{1} p_{2} p_{3}\right)=\frac{g^{2} M_{Z}\left(U_{11} \cos \eta+U_{12} \sin \eta\right)}{\left[\left(p_{1}+p_{2}\right)^{2}-M_{Z}^{2}+i \Gamma_{Z} M_{Z}\right]} \times \\
& {\left[2\left(p_{1 \mu_{2}} g_{\mu_{1} \mu_{3}}-p_{2 \mu_{1}} g_{\mu_{2} \mu_{3}}\right)-\left(p_{1}-p_{2}\right)_{\mu_{3}} g_{\mu_{1} \mu_{2}}\right] \text {, }} \\
& \mathcal{F}_{\mu_{1} \mu_{2} \mu_{3}}^{(-)}\left(p_{1} p_{2} p_{3}\right)=\frac{i g^{2} M_{W}}{\cos \theta_{w}} g_{\mu_{1} \mu_{2}}\left(p_{1}+p_{2}+p_{3}\right)_{\mu_{3}} \mathcal{A}\left(p_{1} \cdot p_{2}\right) \\
& \mathcal{A}\left(p_{1} \cdot p_{2}\right)=\sum_{j=1}^{3} A_{j}\left[\left(p_{1}+p_{2}\right)^{2}-M_{j}^{2}+i \Gamma_{j} M_{j}\right]^{-1}, \\
& A_{j}=U_{13}\left[\cos \eta \sin \eta\left(U_{j 1}^{2}-U_{j 2}^{2}\right)-\cos (2 \eta) U_{j 1} U_{j 2}\right]+ \\
& U_{12}\left[\cos ^{2} \eta U_{j 1} U_{j 2}+\cos \eta \sin \eta U_{j 2} U_{j 3}\right]-U_{11}\left[\cos \eta \sin \eta U_{j 1} U_{j 3}+\sin ^{2} \eta U_{j 2} U_{j 3}\right] .
\end{aligned}
$$

$M_{j}$ are the masses of the three physical scalars $\left(M_{j}=M_{j}\left(\xi_{*}\right) \simeq M_{j}(0)\right), \Gamma_{j}$ are the corresponding widths, and $\eta=(\beta-\alpha)$. In this particular case, we explicitly see that $\left|T_{+}\right|^{2}$ and $\left|T_{-}\right|^{2}$ are symmetric under $p_{1} \leftrightarrow p_{2}$, while $\left(T_{+}^{*} T_{-}+T_{+} T_{-}^{*}\right)$ is antisymmetric (summation over the final helicities $h_{j}$ is always assumed). Therefore, $\left|T_{+}\right|^{2}$ and $\left|T_{-}\right|^{2}$ contribute to $\Gamma$ and not to $\Gamma_{f b}$, while $\left(T_{+}^{*} T_{-}+T_{+} T_{-}^{*}\right)$ contributes to $\Gamma_{f b}$ and not to $\Gamma$ (for the decay $H^{o} \rightarrow W^{+} W^{-} Z$ ). Hence, we see explicitly that relation (11) holds in the specific discussed case.

In the further calculation, we will assume that $M_{1}\left(=M_{H^{\circ}}\right)>\left(2 M_{W}+M_{Z}\right)$ and $M_{2}, M_{3}<$ $2 M_{W}$, and that $\Gamma_{2}$ and $\Gamma_{3}$ are consequently negligible $\left(\Gamma_{2}, \Gamma_{3} \ll \Gamma_{Z} \simeq 2.5 \mathrm{GeV}\right)$. The asymmetry signal $\Gamma_{f b}$ would then be proportional to $\Gamma_{W}$ and $\Gamma_{Z}$. Furthermore, we will assume $\xi<1$ and $\xi_{*}=\left(\lambda_{5}-\lambda_{6}\right) \xi<1$, in order to use the expressions (6). Then it follows

$$
\mathcal{A}\left(p_{1} \cdot p_{2}\right) \simeq \frac{\left(A_{l=2}\right)\left(M_{2}^{2}-M_{3}^{2}\right)}{\left[\left(p_{1}+p_{2}\right)^{2}-M_{2}^{2}\right]\left[\left(p_{1}+p_{2}\right)^{2}-M_{3}^{2}\right]}\left[1+\mathcal{O}\left(\xi \xi_{*}\right)\right]
$$




$$
\Gamma_{f b}\left(H^{o} \rightarrow W^{+} W^{-} Z\right) \simeq \Delta \times\left(\cos \delta \cos \eta \sin ^{2} \eta\right) \xi_{*}\left[1+\mathcal{O}\left(\xi \xi_{*}\right)\right]
$$

The width $\Delta$ in the above formula 1 is

$$
\Delta=\frac{M_{W}^{2} v^{2} g^{4}}{M_{H^{o}}^{3} 2^{8} \pi^{3} \cos ^{2} \theta_{w}} \times I\left(\left\{\frac{M_{j}}{M_{W}}\right\}\right) \simeq \frac{2700 G e V^{4}}{M_{H^{o}}^{3}} \times I,
$$

where $I\left(\left\{M_{j} / M_{W}\right\}\right)$ is a specific "forward-backward" asymmetry integral on the corresponding Dalitz plot, containing suppression factors $\Gamma_{W} / M_{W}$ and $\Gamma_{Z} / M_{Z}$. Numerical calculations yield for $M_{1}\left(=M_{H^{\circ}}\right)=400-800 \mathrm{GeV}$ and $M_{2}, M_{3}=0-100 \mathrm{GeV}: 0.23 \lesssim I \lesssim 90$. The corresponding values for $\Delta$ are given in Table 1. If assuming $0.2<\xi_{*}(<1)$ and $\cos \delta \cos \eta \sin ^{2} \eta \gtrsim 0.5$, (16) gives

$$
\Gamma_{f b}\left(H^{o} \rightarrow W^{+} W^{-} Z\right) \gtrsim(0.1) \Delta
$$

We may also construct the dimensionless asymmetry parameter

$$
\mathcal{A}_{f b}=\frac{\Gamma_{f b}\left(H^{o} \rightarrow W^{+} W^{-} Z\right)}{\Gamma\left(H^{o} \rightarrow W^{+} W^{-} Z\right)}=\frac{N_{f b}}{N} \simeq \rho \frac{\cos \delta \sin ^{2} \eta}{\cos \eta} \xi_{*}\left[1+\mathcal{O}\left(\xi \xi_{*}\right)\right]
$$

The dimensionless parameter $\rho$ is small $\left(\mathcal{O}\left(10^{-3}\right)\right)$, due to the suppression factors $\Gamma_{W} / M_{W}$ and $\Gamma_{Z} / M_{Z}$, and its values are also included in Table 1.

Here we have to mention that the relation (18) is not valid in the limiting case of $\cos \eta \rightarrow 0$, because in such a case $\Gamma\left(H^{o} \rightarrow W^{+} W^{-} Z\right) \simeq 0+\mathcal{O}\left(\xi_{*}{ }^{2}\right)$. In such a case, $\mathcal{A}_{f b}$ could be large $(\lesssim \mathcal{O}(1))$.

For completeness, we also write the decay width $\Gamma\left(H^{o} \rightarrow W^{+} W^{-} Z\right)$ in the theory discussed here

$$
\Gamma\left(H^{o} \rightarrow W^{+} W^{-} Z\right)=\Gamma_{+}+\Gamma_{-},
$$

where

$$
\begin{aligned}
\Gamma_{+}= & \Gamma\left(H^{o} \rightarrow\left(W^{+} W^{-} Z\right)_{C P=+1}\right)=\left(U_{11} \cos \eta+U_{12} \sin \eta\right)^{2} \Gamma^{M S M}\left(H^{o} \rightarrow W^{+} W^{-} Z\right) \\
\simeq & \cos ^{2} \eta \Gamma^{M S M}\left(H^{o} \rightarrow W^{+} W^{-} Z\right)\left[1+\mathcal{O}\left(\xi \xi_{*}\right)\right] \\
& \Gamma_{-}=\Gamma\left(H^{o} \rightarrow\left(W^{+} W^{-} Z\right)_{C P=-1}\right) \simeq\left(\cos ^{2} \delta \sin ^{4} \eta\right) \xi_{*}^{2} G\left[1+\mathcal{O}\left(\xi^{2}\right)\right],
\end{aligned}
$$

\footnotetext{
${ }^{4}$ Strictly speaking, $\Gamma_{f b} \simeq \Delta \times\left(\cos \delta \cos \eta \sin ^{2} \eta\right)\left[1+\frac{M_{2}^{2}-M_{3}^{2}}{M_{1}^{2}-M_{3}^{2}} \tan \delta \cot \eta\right] \xi_{*}+\mathcal{O}\left(\xi \xi_{*}{ }^{2} \Delta\right)$, $(\delta=\beta+\alpha, \eta=\beta-\alpha)$, which reduces to the above form for $M_{2}^{2}, M_{3}^{2} \ll M_{1}^{2}$, or for $M_{2}=M_{3}$.
} 
where the widths $G$, as well as $\Gamma^{M S M}\left(H^{o} \rightarrow W^{+} W^{-} Z\right)$ of the minimal SM, are given in Table 2 for various values of the scalar masses 0 . Note that $\Gamma_{+}$is the contribution from diagrams of Figs. 1a,1b $\left(\propto\left|T_{+}\right|^{2}\right)$, and $\Gamma_{-}$from the diagram of Fig. $2\left(\propto\left|T_{-}\right|^{2}\right)$. Here we see that the width $\Gamma\left(H^{o} \rightarrow W^{+} W^{-} Z\right)$ is not substantially affected by small $\xi_{*}$ parameter. Anyway, this width does not provide an experimental signature for detecting CP-violation. $\Gamma^{M S M}$ for this decay have also been calculated by other authors ( [7], [8], and references therein).

We find that the parameter $\Gamma_{f b}$ (eq. (10)) and the related $\mathcal{A}_{f b}$ (eq. (18)) are possibly relevant in general for experimental investigations of purely bosonic CP-violation effects. We calculated this quantity within the minimal extension of the SM (two Higgs doublets), and found that $\Gamma_{f b}$ may be appreciable, although in general much smaller than $\Gamma\left(H^{o} \rightarrow W^{+} W^{-} Z\right)$. Interestingly enough, the final state interactions do not represent any problem, i.e. they do not give any spurious (CP-conserving) contributions to $\Gamma_{f b}$. On the other hand, the final state interactions (dominated by $W$ and $Z$-width) are in fact crucial, together with $\xi_{*} \neq 0$, for the nonzero CP-violation signal $\Gamma_{f b} \neq 0$. Furthermore, the CP-violation coming from the CKMmatrix would not give any contribution to CP-violating effects for the considered decay at the tree or 1-loop level, but possibly only at the 2-loop level, and can be therefore safely ignored.

LHC and SSC would be able to produce Higgs with mass of several hundred GeV (if such a Higgs exists), mostly through the gluon fusion mechanism [9] and the intermediate boson fusion mechanism [10]. Taking the yearly estimated event rate at LHC for the integrated luminosity to be $10^{41} \mathrm{~cm}^{-2}\left(10^{40} \mathrm{~cm}^{-2}\right.$ at SSC), we expect roughly $10^{3}$ events $H^{o} \rightarrow W^{+} W^{-} Z$ per year [.

Several sources of background would pose a problem for identifying such events - particularly the direct production of $W^{+} W^{-} Z\left(p \bar{p} \rightarrow W^{+} W^{-} Z\right)$ and the QCD continuum $(p \bar{p} \rightarrow$ $Z+4 j e t s)$. It has been argued [7] that the background effects of the direct production would not be a major problem for measuring $\Gamma\left(H^{o} \rightarrow W^{+} W^{-} Z\right)$ at SSC for $M_{H} \approx 500-600 G e V$.

However, $\Gamma_{f b}\left(H^{o} \rightarrow W^{+} W^{-} Z\right)$ is such a difference of the widths for which any background effects of the direct production that do not violate CP-symmetry are cancelled out. To see this, we must recall that in the "forward-backward" difference of events $N_{f b}\left(H^{o} \rightarrow W^{+} W^{-} Z\right)\left(\propto \Gamma_{f b}\right)$ we make the sum (average) over the polarizations of the incoming constituent particles (unpo-

\footnotetext{
${ }^{5}$ Note that the angle $\eta=\beta-\alpha$ may be obtained from experiments measuring the $W^{+}-W^{-}$-Higgs couplings. The angle $\beta$ may be restricted indirectly by experiments whose results depend on the ratio of the vacuum expectation values, within the considered theory.

${ }^{6}$ In ref. [7, the numbers $N$ of decays are given for the minimal SM, but can be used also in the present model as order-of-magnitude estimates.
} 
larized). For the case of direct production we choose the spin basis $\left|S, S_{z}\right\rangle$ for polarizations of the initial $q \bar{q}$ (or $g g$ ) states \. These initial states have well-defined CP $\left(C P(q \bar{q})=(-1)^{S_{q \bar{q}}+1}\right.$, $\left.C P(g g)=(-1)^{S_{g g}}\right)$, and hence also the resulting directly produced $W^{+} W^{-} Z$ states would have the same well-defined CP, provided the direct production processes themselves do not contain appreciable CP-violating vertices. Therefore, such events would contribute zero to $N_{f b}\left(W^{+} W^{-} Z\right)\left(\propto \Gamma_{f b}\left(W^{+} W^{-} Z\right)\right)$. This argument also holds if the initial $q \bar{q}$ have opposite polarizations $(S=1)$. The CKM-type CP-violation effects in the direct production could in principle contribute to $N_{f b}$, but their effects are very small for $q \bar{q}(q=u, d)$ and $g g$ initial states (CP-violating asymmetries $\left.\lesssim \mathcal{O}\left(10^{-6}\right)\right)$ [1].

Most of the QCD continuum background may be eliminated with on-mass-shell constraints and certain additional cuts [0]. However, several aspects of this problem remain open, and this background may pose a problem for determining $\Gamma_{f b}$.

It is possible that the CP-violating phase $\xi_{*}$ also enters the general Yukawa couplings, specifically the coupling between $H^{o}$ and the top quark. Within the latter scenario, D. Chang and W.-Y. Keung 12 have recently investigated CP-violating asymmetries for the decays $H^{o} \rightarrow W^{+} W^{-}, t \bar{t}$, where the source of the asymmetries is the imaginary part $\left(\propto \xi_{*}\right)$ of the Yukawa coupling of $H^{o}$ to the top quark. They concluded that such asymmetries can be measurable in future colliders such as SSC or LHC. These asymmetries were $\mathcal{A}\left(H^{o} \rightarrow W^{+} W^{-}\right)=$ $\left(N\left(W_{L}^{+} W_{L}^{-}\right)-N\left(W_{R}^{+} W_{R}^{-}\right)\right) / N\left(W^{+} W^{-}\right)$and $\mathcal{A}\left(H^{o} \rightarrow t \bar{t}\right)=\left(N\left(t_{L} \bar{t}_{L}\right)-N\left(t_{R} \bar{t}_{R}\right)\right) / N(t \bar{t})$, where $L$ and $R$ stand for helicities $-1,+1$, respectively. They found out that $\mathcal{A}\left(H^{o} \rightarrow W^{+} W^{-}\right) \lesssim$ $\mathcal{O}\left(10^{-3}\right)$ and $\mathcal{A}\left(H^{o} \rightarrow t \bar{t}\right) \lesssim \mathcal{O}\left(10^{-1}\right)$, for $m_{t} \approx 150 \mathrm{GeV}$. Furthermore, the branching ratios for these decays are larger by one order of magnitude than those for $H^{o} \rightarrow W^{+} W^{-} Z$. For the latter decay, $\mathcal{A}_{f b}$ in most cases does not exceed $\mathcal{O}\left(10^{-3}\right)$, according to eq. (18) and Table 1 . On the other hand, the helicity asymmetries $\mathcal{A}\left(H^{o} \rightarrow W^{+} W^{-}\right), \mathcal{A}\left(H^{o} \rightarrow t \bar{t}\right)$ cannot be measured directly, but have to be decoded from the asymmetry of the energy distributions of the resulting final leptons. It appears that roughly one order of magnitude is lost due to this decoding, i.e. $\mathcal{A}_{l^{+} l^{-}}=\left(\left\langle E_{l^{-}}\right\rangle-\left\langle E_{l^{+}}\right\rangle\right) /\left\langle E_{l^{+}}\right\rangle$are about one order of magnitude smaller than the corresponding $\mathcal{A}\left(H^{o} \rightarrow W^{+} W^{-}\right), \mathcal{A}\left(H^{o} \rightarrow t \bar{t}\right)$ (the decoding for $H^{o} \rightarrow Z Z$ is even harder). Hence, comparing the results of ref. [12] with the results of the present paper, we conclude that the measurability of $\mathcal{A}\left(H^{o} \rightarrow W^{+} W^{-}\right)$(and the problems connected with it) is comparable to the measurability of $\mathcal{A}_{f b}\left(H^{o} \rightarrow W^{+} W^{-} Z\right)$, while the measurement of $\mathcal{A}\left(H^{o} \rightarrow t \bar{t}\right)$ (for $m_{t} \approx 150 \mathrm{GeV}$ ) clearly

\footnotetext{
${ }^{7}$ We are allowed to take any convenient polarization basis, since at the end we sum over all initial polarizations.
} 
appears to be more promising.

One major problem in measuring $\Gamma_{f b}\left(\right.$ or $\left.\mathcal{A}_{f b}\right)$ would be a somewhat low production rate of heavy Higgs at SSC and $\operatorname{LHC}\left(N \sim 10^{3}\right.$ decays $H^{o} \rightarrow W^{+} W^{-} Z$ per year $)$. Since $\mathcal{A}_{f b}\left(H^{o} \rightarrow W^{+} W^{-} Z\right)\left(=\left(N_{\text {forw }}-N_{\text {backw. }}\right) / N\right)$, in the framework discussed here, is in most cases not exceeding $\mathcal{O}\left(10^{-3}\right)$ (eq. (18) and Table 1), many years of measurements would be needed to obtain possibly statistically significant effects. Nonetheless, we believe that the proposed quantity $\Gamma_{f b}$ (or $\mathcal{A}_{f b}$ ), or related quantities, may eventually become relevant for experimental tests of CP-violation of the purely bosonic sector. Furthermore, the $\Gamma_{f b}$ and $\mathcal{A}_{f b}$ parameters should be investigated numerically also for the case of larger $\xi_{*}$ parameter of CP-violation, and they may be substantially larger in this case.

\section{Acknowledgement}

The author would like to thank K.J. Abraham, M. Nowakowski, E.A. Paschos, A. Pilaftsis and Y.-L. Wu for helpful discussions, particularly concerning the possible CKM-type of CPviolation effects. The author wishes to thank the Deutsche Forschungsgemeinschaft (DFG) and the CEC Science project No. SC1-CT91-0729 for financial support during the progress of this work. 


\section{References}

[1] S. Weinberg, Phys. Rev. Lett. 63 (1989) 2333, Phys. Rev. D42 (1990) 860; A. Mendez and A. Pomarol, Phys. Lett. B272 (1991) 313; W. Bernreuther, T. Schröder and T. N. Pham, Phys. Lett. B279 (1992) 389; D. Atwood et al., Technion preprint Technion-PH-92-38; B. Grządkowski and G.F. Gunion, preprint UCD-92-18; B. Grządkowski, J.F. Gunion and T.C. Yuan, preprint UCD-93-2

[2] A. Cohen, D. Kaplan and A. Nelson, Phys. Lett. B263 (1991) 86

[3] N. Turok and J. Zadrozny, Nucl. Phys. B358 (1991) 471; L. McLerran, M. Shaposhnikov, N. Turok and M. Voloshin, Phys. Lett. B256 (1991) 451

[4] J. F. Gunion, H. E. Haber, G. Kane and S. Dawson, "The Higgs Hunters's Guide", p. 195 (Addison-Wesley Publishing Co.,1990), and Errata, preprint SCIPP-92/58 (Dec. 1992); note that the $\lambda_{7}$-term mentioned in the Errata can be eliminated also in the case of $\mathrm{CP}_{-}$ violation, by redefining the parameters $\lambda_{j}(\mathrm{j}=5,6,7), \xi$, and the phase of $\Phi_{2}$ appropriately; the obtained potential has then again the form of eq. (1)

[5] G. Cvetič, M. Nowakowski and A. Pilaftsis, Phys. Lett. B301 (1993) 77

[6] J. Werle, "Relativistic Theory of Reactions" (North-Holland Publ. Company, Amsterdam, 1966)

[7] P. Langacker and J. Liu, Phys. Rev. D46 (1992) 5069

[8] R. Decker, M. Nowakowski and A. Pilaftsis, Z. Phys. C57 (1993) 339

[9] H. Georgi, S. L. Glashow, M. E. Machacek and D. V. Nanopoulos, Phys. Rev. Lett. 40 (1978) 692

[10] S. Petcov and D. T. T. Jones, Phys. Lett. B84 (1979) 440; R. N. Cahn and S. Dawson, Phys. Lett. 136B (1984) 196

[11] M. Nowakowski and A. Pilaftsis, Mod. Phys. Lett. A4 (1989) 821, Z. Phys. C42 (1989) 449

[12] D. Chang and W.-Y. Keung, Phys. Lett. B305 (1993) 261 


\section{Tables}

Table 1

\begin{tabular}{|c||c|c|c||c|c|c|}
\hline$M_{H^{o}}$ & $\Delta^{(1)}$ & $\Delta^{(2)}$ & $\Delta^{(3)}$ & $\rho^{(1)} \cdot 10^{3}$ & $\rho^{(2)} \cdot 10^{3}$ & $\rho^{(3)} \cdot 10^{3}$ \\
\hline 300 & 0.26 & 0.38 & 0.56 & 0.98 & 1.44 & 2.10 \\
\hline 400 & 9.8 & 13.0 & 17.2 & 1.49 & 2.00 & 2.65 \\
\hline 500 & 40.5 & 52.0 & 67.0 & 1.16 & 1.50 & 1.92 \\
\hline 600 & 101. & 124. & 155. & 0.91 & 1.12 & 1.40 \\
\hline 700 & 196. & 236. & 300. & 0.73 & 0.88 & 1.10 \\
\hline 800 & 330. & 400. & 492. & 0.61 & 0.74 & 0.91 \\
\hline
\end{tabular}

Table 1: $\Delta$ widths (in $k e V$ ) and $\rho$ numbers (eqs. (17),(18)) for various values (in $G e V$ ) of the heavy Higgs mass $M_{H^{\circ}}\left(=M_{1}\right)$. The superscripts (1), (2), (3) denote these values for the cases when the masses $\left(M_{2}, M_{3}\right)$ of the other two physical scalars (in $G e V$ ) are $(0,0),(100,0)$ or $(0,100)$, and $(100,100)$, respectively. 
Table 2

\begin{tabular}{|c||c|c|c||c|}
\hline$M_{H^{o}}$ & $G^{(1)}$ & $G^{(2)}$ & $G^{(3)}$ & $\Gamma^{M S M}\left(H^{o} \rightarrow W W Z\right)$ \\
\hline 300 & $2.3 \cdot 10^{-4}$ & $5.0 \cdot 10^{-4}$ & $1.12 \cdot 10^{-3}$ & $1.16 \cdot 10^{-3}$ \\
\hline 400 & $5.0 \cdot 10^{-3}$ & $9.4 \cdot 10^{-3}$ & $1.84 \cdot 10^{-2}$ & $2.88 \cdot 10^{-2}$ \\
\hline 500 & $2.04 \cdot 10^{-2}$ & $3.6 \cdot 10^{-2}$ & $6.5 \cdot 10^{-2}$ & $1.54 \cdot 10^{-1}$ \\
\hline 600 & $5.15 \cdot 10^{-2}$ & $8.6 \cdot 10^{-2}$ & 0.152 & 0.490 \\
\hline 700 & 0.103 & 0.167 & 0.287 & 1.18 \\
\hline 800 & 0.179 & 0.285 & 0.480 & 2.39 \\
\hline
\end{tabular}

Table 2: $G$ widths (eq. (20)), and the decay width $\Gamma^{M S M}$ of the minimal SM, for various values of the Higgs mass. All values are in $G e V$. The superscripts of $G$ have the same meaning as those in Table 1. 